\title{
LHC Experiments Communication and Engagement with New Audiences
}

\author{
Despina Hatzifotiadou ${ }^{* \dagger}$ \\ Universita e INFN, Bologna (IT) \\ E-mail: despina.hatzifotiadou@cern.ch
}

\begin{abstract}
During the last decades, with the development of a multitude of communication platforms, social media and other offerings of information technology, communication has acquired a very important role in our life. Thus the necessity and importance of science communication keeps increasing and has unavoidably become part of the scientific process. In this paper we first discuss briefly the targets of our communication strategy, the objectives and the messages. We then present a non-exhaustive list and some examples of communication methods and platforms used by the four big LHC experiments: ALICE, ATLAS, CMS and LHCb. Emphasis is given on engagement with new audiences.
\end{abstract}

Fourth Annual Large Hadron Collider Physics

13-18 June 2016

Lund, Sweden

\footnotetext{
* Speaker.

† On behalf of ALICE, ATLAS, CMS and LHCb collaborations.
} 


\section{Introduction}

Communication is an inseparable part of researchers' work. Our targets are a multitude of audiences: the general public, school children - both in elementary and secondary education - and their teachers, as well as the broader scientific community. Some more special categories are the media (journalists, reporters etc) and the stakeholders (funding agencies and politicians).

Demystifying science is one of our main aims. The myth of inapproachable scientists who, cut off from real life, in their ivory tower, spend public money doing things that no one understands, does not hold any longer. The public should not be in awe of the world of scientific research. It is the duty of scientists to be open about what they are doing and offer to the public as much information as possible and as often as possible. For one thing, they have the right to know how their taxes are used. Direct consequence of this will be an increase of the scientific literacy of the public. Being aware of what science is about will also help the general public appreciate scientific research. Communication with pupils aims to inspire them, to stimulate their curiosity and motivate them, by giving them opportunities to directly engage with the world of science. Inspiring teachers is equally if not more important, since they propagate the love of science and are thus a strong multiplication factor. The same applies to journalists who have an incredible power over the public through press, television and online media. Informing the stakeholders about our work and especially our results is also essential; it helps ensure funding and support.

The messages delivered are many different ones. One of our priorities is to convince the public about the necessity of scientific research for society; to convey the fact that pure science, seeking answers to fundamental questions and not geared towards applications, is indispensable and not a luxury; and that this quest for knowledge, driven by curiosity, which is part of our human nature, leads to applications improving our quality of life. We want to explain the impact of science on society, especially when it comes to developments in technology and medicine.

In our effort to lift the veil that covers scientific research, we try to explain our methods, show the instruments we use and obviously explain our results.

Sharing our enthusiasm and love of science, and our excitement when we are lucky enough to make ground-breaking discoveries, we hope to inspire the youth, the next generation of scientists.

Another message that we want to convey is that science is for all and not only for the elected few. We try to emphasize the importance of diversity, be it cultural, religious, political etc. CERN and the LHC experiments' collaborations, bringing together scientists, engineers, technicians and students from about hundred different nationalities, is an indisputable example of what such a diverse community can achieve. In an effort to increase female participation in the scientific and technological sector, we try to avoid presenting the stereotype of the male scientist; instead, we emphasize female presence and show female role models.

\section{The methods and tools}

A variety of methods and tools, using different platforms, are used for communication and for engaging with new audiences.

Some of the "traditional" communication tools are exhibitions, and the methods used include visits and public talks, tailored to the audience's specific level and needs (general public, students, 
media). Special events are also organised with the participation of the LHC collaborations (European Researchers' Night on the last Friday in September every year; Open Days, Science Fairs, etc.).

The big variety of web-based tools that have become available in the last decade or so tend to dominate the communications landscape. Social media (Facebook, Twitter, Google+, etc.) are an example, blogs and online newsletters is another, web pages are a source of a wealth of information. The platforms and the equipment used for video-conferencing, which is nowadays a very common method of communication among scientists, have inspired virtual visits.

Various hands-on and educational activities are another way of engaging with the public; a very successful such activity is masterclasses in particle physics [1]. More than 10000 high-school students every year are introduced to the wonders of particle physics and have the opportunity to analyse real data from the LHC experiments, thus being directly involved with the world and the methods of research (see presentation on Masterclasses in the same session at this conference [2]). A variety of platforms and tools make it possible for all interested to have a glimpse at the LHC data and do their own analysis. The CERN Open Data Portal is an example [3]; another example is Citizen Science launched by the ATLAS experiment with a number of different projects (ATLAS@ @ome, Higgs Hunters, Higgs Machine Learning) [4],[5].

In what follows we will mainly concentrate on the online communication tools.

\section{Public web pages, blogs, newsletters}

In addition to the collaboration web pages, restricted to their members, all LHC experiments have web pages where access is free, with a plethora of information for the public.

The links to the public web pages of the four big LHC experiments are given in the Appendix.

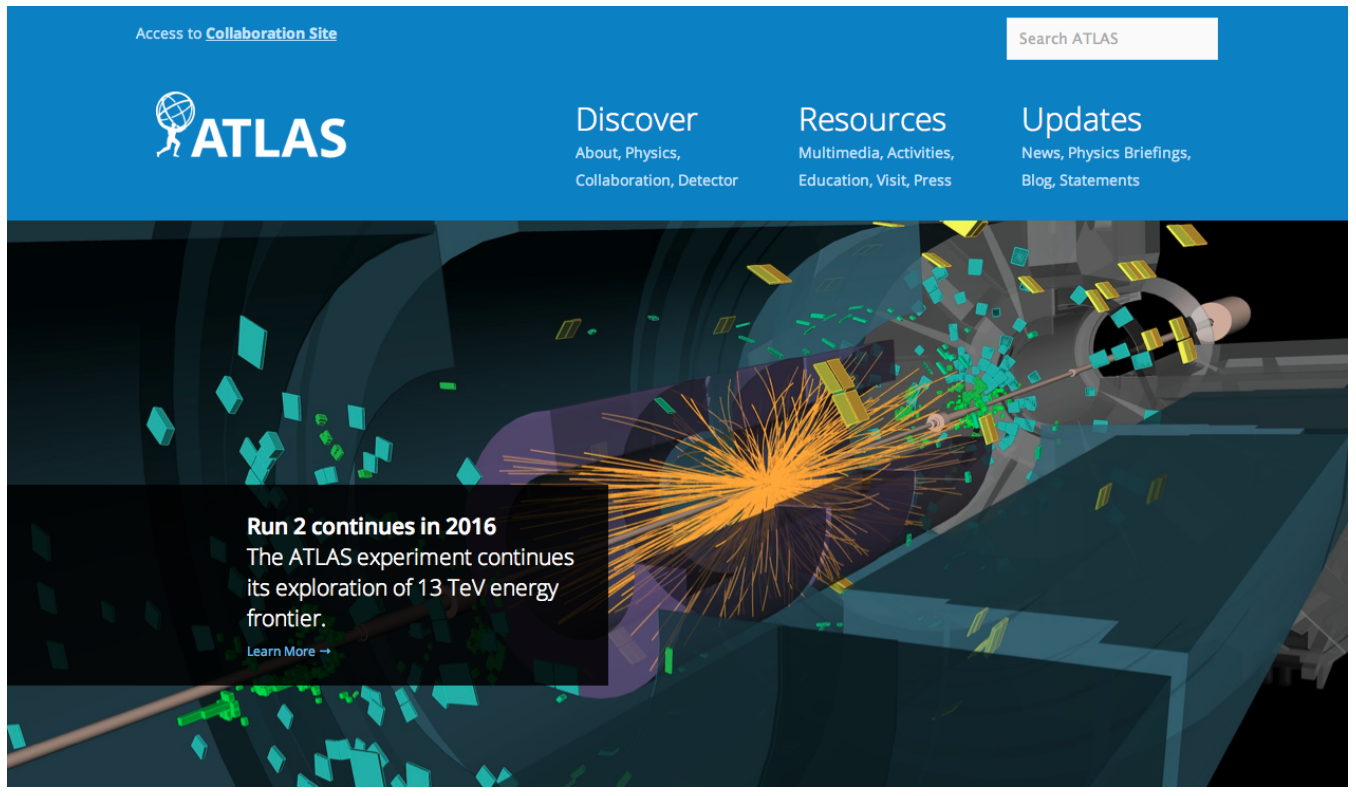

Figure 1: ATLAS public web page 
Some of them were designed years ago and are updated whereas others have been made new.

As an example, the newly released ATLAS public pages are presented here, shown in Figure 1. They were developed after audience survey and were designed by a professional company [6]; they use Drupal, the CERN standard content management system. The static information is organized in two chapters, under the titles "Discover" and "Resources". The content under "Updates" is obviously dynamic: ATLAS news, physics briefings, statements and blogs are regularly posted there.

The LHCb collaboration posts news and recent physics results on the first page of their public web pages; so does the CMS collaboration, under two different categories.

The ALICE collaboration issues ALICE Matters (http://alicematters.web.cern.ch/), an online monthly newsletter, with news from the collaboration, the detectors and the data-taking, features on physics topics and conferences, and interviews of ALICE members.

\section{Social media}

All LHC collaborations use social media, and they have "official" Facebook, Google+ and Twitter accounts; the links are given in the Appendix.

News from the experiments or news of general interest from LHC, CERN and the particle physics community are posted on the above social media. Given that these accounts have thousands or tens of thousands of "likes" or followers, it is clear that social media are a very powerful means of communication, reaching audiences all over the globe. Individuals also often use their personal accounts as a means of communicating science to their followers.

The CMS experiment has recently launched a twitter account called @CMSVoices, which is controlled by a different CMS member every month. This regular change of the person who tweets allows to show the different flavours and colours in a large collaboration. It also enables members of the public to engage directly with a real person from CMS instead of tweeting @ CMSexperiment without getting any response.

Youtube is undoubtedly a very widely used communication channel; numerous videos can be found on the channels of the LHC collaborations; the links are given in the Appendix.

They contain educational material, simplified explanations of physics topics, interviews with physicists, filming from detector installation, to name but a few. An example of a very successful video is "Flying over ALICE" (https://www.youtube.com/watch?v=yWBWzIUCNpw), a film done with a drone flying over the ALICE site at LHC-Point 2, down in the shaft, in the cavern and over the L3 magnet and the muon spectrometer. Once it was released on youtube and announced on social media, within days it was on many online news sites and in a period of 12 days it had more than 18000 viewings, whereas by now it has exceeded 64000 viewings.

\section{Virtual visits}

Virtual visits are a novel way of reaching out to remote audiences who do not have the opportunity to visit CERN physically due to geographical, economical or other limitations. A virtual visit is basically a videoconference, which, if appropriate equipment is available, can also be recorded and webcast. ATLAS virtual visits are conducted from the ATLAS Control Room, where there is 
special equipment for this purpose. The virtual guide is a physicist speaking the language of the virtual visitors who, in most cases, are high-school students; virtual visits are also organized in conjunction with local events, such as Science Festivals, Open Days etc.

The virtual visit includes a brief introduction to CERN, LHC and ATLAS followed by a virtual tour of the control room and explanation of the shifters' roles. This is followed by a question and answer session, and it is actually this interaction between remote audience and physicists involved in research which is most valuable.

ATLAS has a web site dedicated to virtual visits, http://cern.ch/atlas-virtual-visit. Upcoming virtual visits are announced there, including a brief description of the event and an embedded viewer for watching the webcast. There is a booking system including tests of the connection before the visit.

The Digital Communication Award 2013 for best online event was awarded to ATLAS outreach for the virtual visits. They were also finalists for the European Excellence Award 2013. By now they have conducted more than 250 virtual visits in 40 countries all over the world.

CMS virtual visits are conducted using portable equipment and typically make a virtual tour of the installations of CMS at LHC-Point 5, including virtual tour of the CMS cavern during shutdown periods; they also have a booking system. More information can be found on the dedicated web site http://cms.web.cern.ch/content/virtual-visits; details on ATLAS and CMS virtual visits in [7].

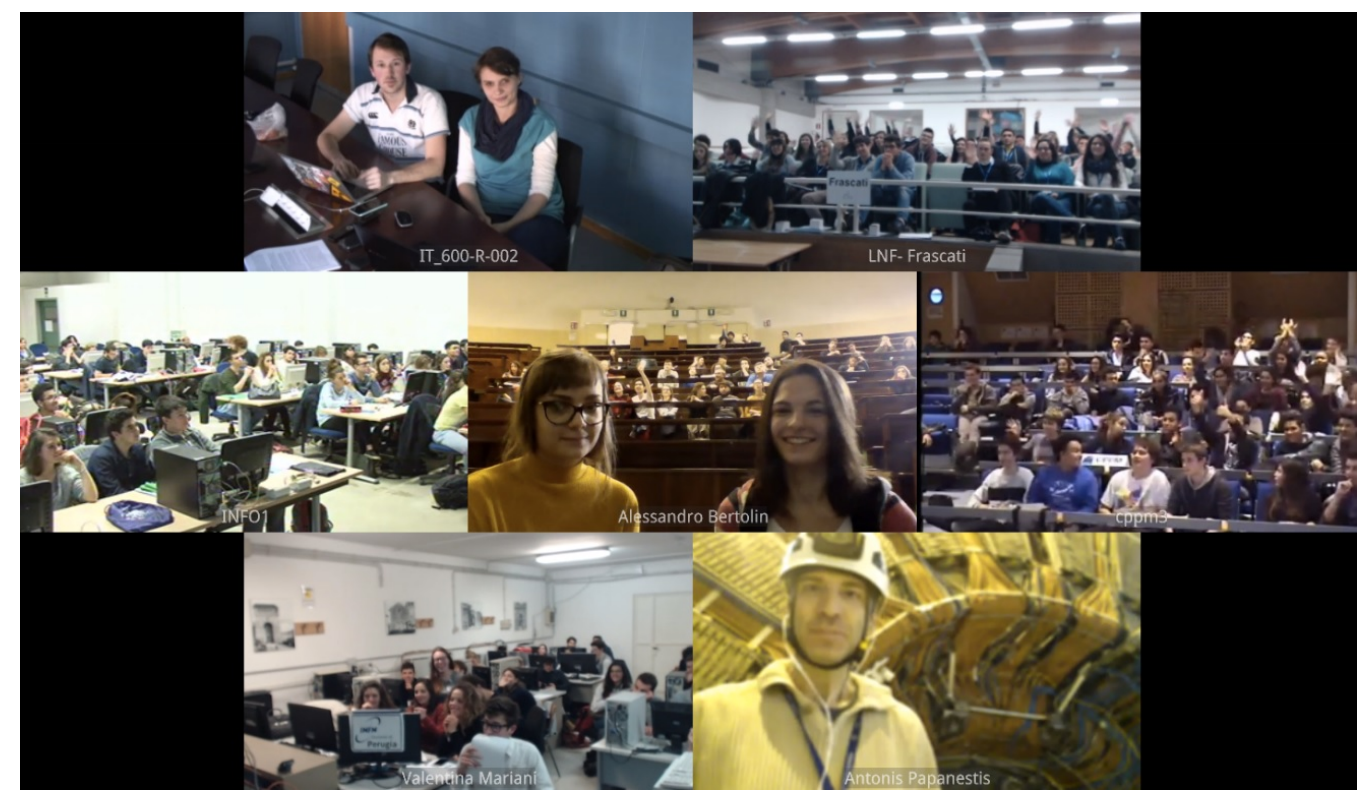

Figure 2: Virtual visit from LHCb Control Room during International Masterclasses 2016.

LHCb have recently equipped the Control Room with video-conference equipment; during International Masterclasses 2016, the moderation session at the end of the day included a virtual visit of the LHCb Control Room as well as the LHCb cavern or the DELPHI experiment. Figure 2 shows such a virtual visit.

ALICE members conduct virtual visits in an ad hoc manner, upon request, using the virtual guide's laptop. They are often combined with local events at the institutes of ALICE members. A 
number of virtual visits have taken place with elementary schools in Greece; it is worth mentioning that, at the end of the virtual visit, the children sang songs and danced to show their gratitude.

All in all, virtual visits is a tool with huge potential since it cancels geographical barriers and allows access to new, remote audiences, who can thus engage with the world of research.

\section{Other activities and tools}

These last years, physicists from the LHC experiments have been organizing, during the Montreux Jazz Festival, sessions with the title "The Physics of Music and the Music of Physics". The festival-goers, a completely new audience, were thus introduced to concepts of particle physics and cosmic rays and had the opportunity to listen to sonification of LHC data, the cosmic piano (music from cosmic muons) and a jazz pianist improvising along with it. In Figure 3 a poster of such a session can be seen.

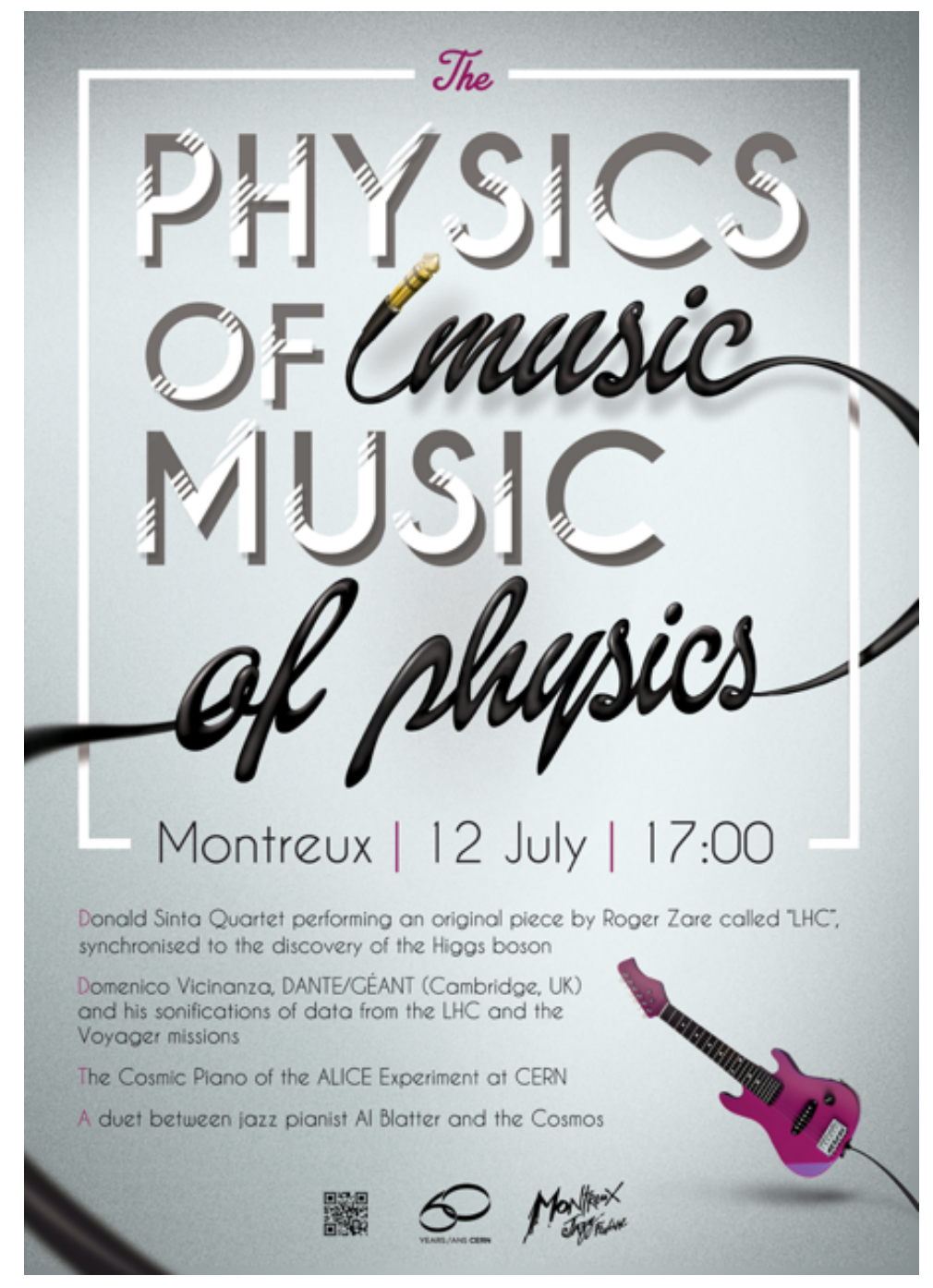

Figure 3: The Physics of Music and the Music of Physics. 
A number of AMA sessions on Reddit were organized at CERN with the participation of LHC physicists. Reddit is an entertainment, social networking and news website. Registered community members can submit content, such as text posts or direct links. AMA (Ask Me Anything) on Reddit are sessions when, during a defined time period reddit users ask questions to a panel. The members of the panel answer. The most recent AMA sessions were on the occasion of the first $13 \mathrm{TeV}$ proton collisions at LHC [8] and when the CMS experiment made publicly available 300 TB of data on the CERN Open Data Portal [9]. This is another way of engaging new audiences (the Reddit users) and giving them the opportunity to interact with physicists.

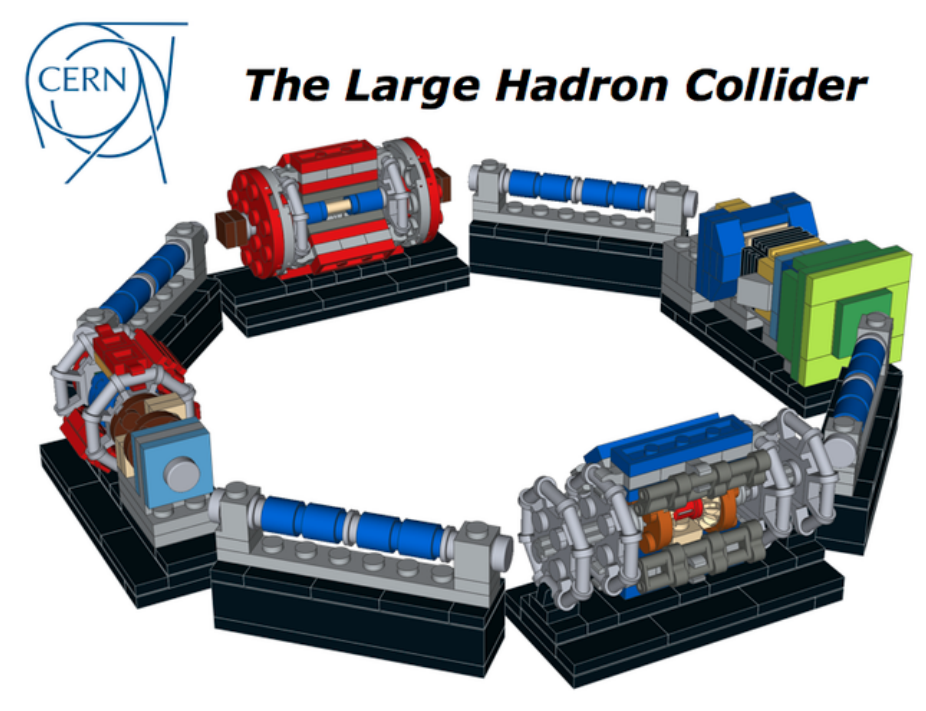

Figure 4: The LHC Lego, submitted to LEGO ideas for voting.

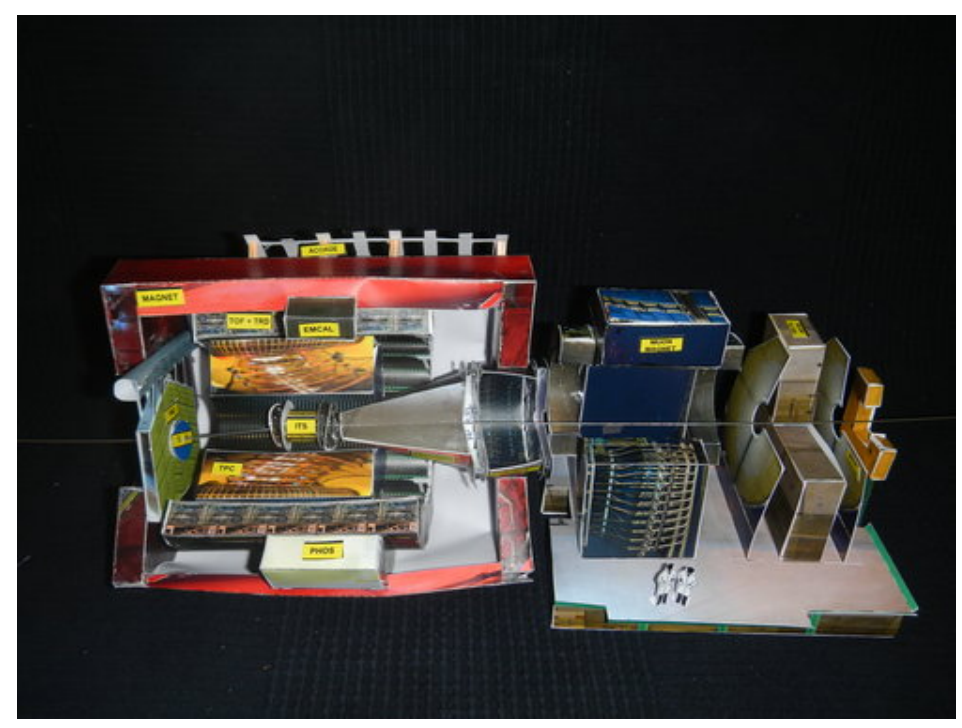

Figure 5: The ALICE papercraft 


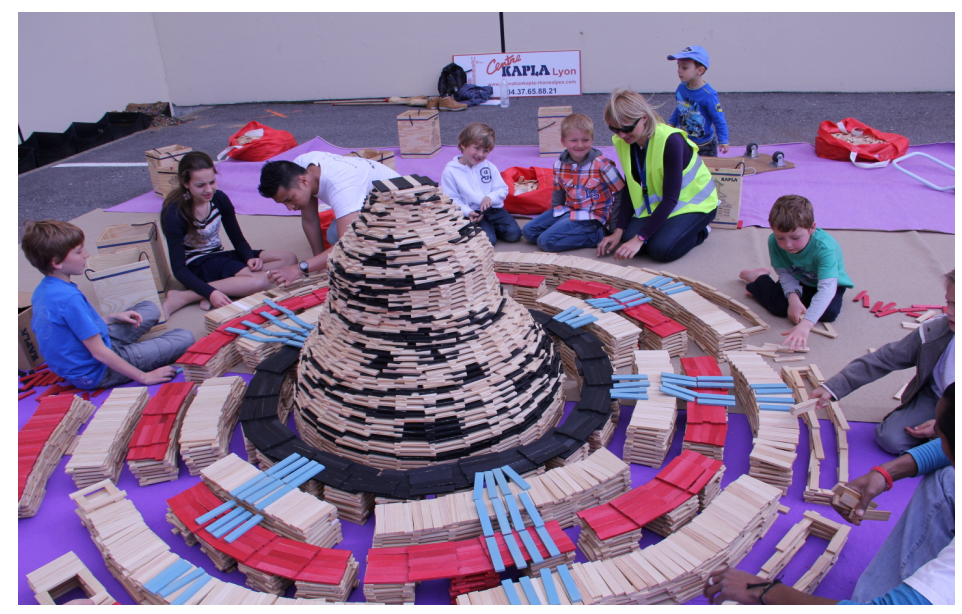

Figure 6: Building CMS out of wooden blocks.

LHC-inspired toys serve as another means of communication, reaching out to children and their parents. The Large Hadron Collider lego, shown in Figure 4, done by an ATLAS Phd student, was put to online voting (lego ideas)[10] and has acquired the 10000 votes needed for LEGO to consider making it into a commercial product, something which would make LHC and its experiments known to much wider audiences. The ALICE papercraft [11], shown in Figure 5, gives the opportunity to children to construct a paper model of the ALICE apparatus: cutting and glueing together all the ALICE subdetectors, they discover all the different components of an LHC experiment. Last but not least, during local events at CMS, children have been encouraged to build the CMS detector out of KAPLA wooden blocks, as shown in Figure 6.

\section{Summary}

As a result of the High Energy Physics community's communication strategy the public has an ever increasing interest in LHC and its experiments; it is clear that online news, blogs and social media fuel further this interest, since they allow people to follow what is happening. Social media in particular have a huge potential for engaging new and wider audiences. The use of the numerous web-based resources offer an invaluable tool for the communication process. Virtual visits are a powerful tool to engage, especially with young people across the globe. It is up to the members of the scientific community to make the best possible use of all tools at their disposal, taking an active role in the communication with the public.

\section{Acknowledgements}

We would like to acknowledge all colleagues from ALICE, ATLAS, CMS, LHCb for offering material and ideas for this presentation; also all members of the LHC Outreach Group (LOG) and the International Particle Physics Outreach Group (IPPOG), as well as the CERN Education, Communications and Outreach group for their contribution in outreach in particle physics. 


\section{Appendix}

Links to the public web pages of the four big LHC experiments:

ALICE http://aliceinfo.cern.ch/Public/Welcome.html

ATLAS http://atlas.cern/

CMS http://cms.web.cern.ch/

LHCb http://lhcb-public.web.cern.ch/lhcb-public/

Links to the "official" Facebook, Google+ and Twitter accounts of the four big LHC experiments:

https://www.facebook.com/ALICE.EXPERIMENT/

https://www.facebook.com/ATLASexperiment/

https://www.facebook.com/CMSexperiment/

https://www.facebook.com/LHCbexperiment/

https://www.twitter.com/ALICEexperiment/

https://www.twitter.com/ATLASexperiment/

https://www.twitter.com/CMSexperiment/

https://www.twitter.com/LHCbexperiment/

https://plus.google.com/+ALICEexperiment/

https://plus.google.com/+ATLASexperiment/

https://plus.google.com/+CMSexperiment/

Youtube channels of the LHC collaborations:

https://www.youtube.com/c/alicematters

https://www.youtube.com/c/cmsexperiment

https://www.youtube.com/user/TheATLASexperiment

\section{References}

[1] http://physicsmasterclasses.org

[2] LHC Masterclasses. Bringing Particle Physics into the Classroom: Present and Future (presentation by Vladimir Gligorov, these Proceedings)

[3] http://opendata.cern.ch/

[4] http://lhcathome.web.cern.ch/

[5] https:www.higgshunters.org/

[6] http://iopscience.iop.org/article/10.1088/1742-6596/664/5/052015

[7] http://pos.sissa.it/cgi-bin/reader/contribution.cgi?id=234/349

[8] http://home.cern/about/updates/2015/05/cern-takes-reddit-discuss-new-energy-frontier

[9] http://home.cern/about/updates/2016/06/cern-hosts-live-reddit-qa-cms-open-data

[10] https://ideas.lego.com/projects/94885

[11] http://alicematters.web.cern.ch/?q=PapercraftOpenDays2013 